\title{
Epigenetics in type 1 diabetes: TNFa gene promoter methylation status in Chilean patients with type 1 diabetes mellitus
}

\author{
Viviana Arroyo-Jousse ${ }^{1}$, Diego F. Garcia-Diaz ${ }^{1}$, Ethel Codner ${ }^{2}$ and Francisco Pérez-Bravo ${ }^{1 *}$ \\ ${ }^{1}$ Nutrigenomics Laboratory, Department of Nutrition, School of Medicine, University of Chile, Av. Independencia 1027, \\ Santiago 8380453, Chile \\ ${ }^{2}$ Institute of Maternal and Child Research, School of Medicine, University of Chile, Av. Santa Rosa 1234, Santiago \\ 8360160, Chile
}

(Submitted 15 March 2016 - Final revision received 9 July 2016 - Accepted 1 October 2016 - First published online 28 November 2016 )

\section{Abstract}

TNF- $\alpha$ is a pro-inflammatory cytokine that is involved in type 1 diabetes (T1D) pathogenesis. The TNFa gene is subject of epigenetic regulation in which folate and homocysteine are important molecules because they participate in the methionine cycle where the most important methyl group donor ( $S$-adenosylmethionine) is formed. We investigated whether TNFa gene promoter methylation status in T1D patients was related to blood folate, homocysteine and TNF- $\alpha$ in a transversal case-control study. We studied T1D patients $(n 25$, mean $=13.7$ years) and healthy control subjects ( $n$ 25, mean $=31 \cdot 1$ years), without T1D and/or other autoimmune diseases or direct family history of these diseases. A blood sample was obtained for determination of serum folate, plasma homocysteine and TNF- $\alpha$ concentrations. Whole blood was used for the extraction of DNA to determine the percentage of methylation by real-time PCR and melting-curve analysis. Results are expressed as means and standard deviations for parametric variables and as median (interquartile range) for non-parametric variables. T1D patients showed a higher TNFa gene promoter methylation (39.2 (sD 19.5) \%) when compared with control subjects (25.4 (sD 13.7) \%) ( $P=0 \cdot 008)$. TNFa gene promoter methylation was positively associated only with homocysteine levels in T1D patients $(r 0.55, P=0 \cdot 007)$, but not in control subjects $(r-0 \cdot 122, P=0 \cdot 872)$. To our knowledge, this is the first work that reports the methylation status of the TNFa gene promoter and its relationship with homocysteine metabolism in Chilean T1D patients without disease complications.

\section{Key words: Epigenetics: Type 1 diabetes: Methylation: TNF- $\alpha$ : Homocysteine}

Type 1 diabetes (T1D) has been defined as a chronic autoimmune disease that appears as a result of total or partial destruction of pancreatic $\beta$-cells ${ }^{(1)}$. About $85 \%$ of new cases of T1D are sporadic ${ }^{(2)}$, affecting children without a family history of the disease. The continuous worldwide rise in T1D incidence $^{(3)}$ cannot be explained by genetics itself, as it has been seen that the number of subjects bearing high-risk genotypes, among recently diagnosed T1D cases, had decreased ${ }^{(4,5)}$. Therefore, this suggests that it is the interaction between environmental and genetic factors that plays a preponderant role in T1D susceptibility ${ }^{(3)}$. In this sense, epigenetics refers to a number of mechanisms by which environmental factors modulate genetic expression through heritable modifications that do not include changes in the DNA sequence ${ }^{(6,7)}$.

DNA methylation, one of these epigenetic mechanisms, is essential for gene expression regulation in mammals. This process involves the covalent addition of a methyl group $\left(\mathrm{CH}_{3}\right)$ to a cytosine in a guanine-cytosine dinucleotide (CpG), from the methyl donor $S$-adenosylmethionine (SAM). The latter is formed in the methionine cycle after folate is reduced and transfers one methyl group to homocysteine (Hcy), a sulfurcontaining amino $\operatorname{acid}^{(8)}$.

Devaraj et al. ${ }^{(9)}$ concluded that T1D corresponds to a pro-inflammatory state after they observed, in T1D patients' monocytes, a higher production of cytokines. CD8+ and CD4+ $\mathrm{T}$ lymphocytes secrete pro-inflammatory cytokines such as TNF- $\alpha$. It is believed that TNF- $\alpha$ could be implicated in pancreatic $\beta$-cell destruction because, in vitro, TNF- $\alpha$, IL- 1 and INF- $\gamma$ have cytotoxic effects upon $\beta$-cells through the production of free radicals, nitric oxide, reactive oxygen species and the activation of apoptosis pathways ${ }^{(10,11)}$.

The importance of epigenetic regulation in the control of TNFa gene expression, during embryonic development (human embryonic stem cell lines $\mathrm{H} 9$ and $\mathrm{H} 1$ ) and in response to LPS (K562, THP-1 and HL60 cell lines and isolated monocytes/ macrophages) ${ }^{(12)}$ or to exogenous TNF- $\alpha$ stimulation $^{(13)}$, has been demonstrated; in all those cases, induced epigenetic changes altered gene expression. Some studies have found differences in the methylation patterns of the TNFa gene promoter $^{(14-16)}$ in different chronic diseases in humans.

Abbreviations: Hcy, homocysteine; T1D, type 1 diabetes.

* Corresponding author: Dr F. Pérez-Bravo, fax +562 27355581, email fperez@med.uchile.cl 
Table 1. Characteristics of study groups

(Means and standard deviations; medians and interquartile ranges)

\begin{tabular}{|c|c|c|c|c|}
\hline \multirow[b]{2}{*}{ Characteristics } & \multicolumn{2}{|c|}{ T1D } & \multicolumn{2}{|c|}{ Controls } \\
\hline & Mean/(median) & SD/(interquartile range) & Mean/(median) & $\mathrm{sD} /$ (interquartile range) \\
\hline$n$ & 25 & - & 25 & - \\
\hline Age (years) & $13 \cdot 7$ & 5.9 & $31 \cdot 1$ & 7.8 \\
\hline $\operatorname{Sex}(F / M)$ & $14 / 11$ & - & $9 / 16$ & - \\
\hline Weight (kg) & $46 \cdot 2$ & $16 \cdot 9$ & 72 & $14 \cdot 1$ \\
\hline Height $(m)$ & $(1.54)$ & $(0.36)$ & $(1 \cdot 70)$ & $(0 \cdot 16)$ \\
\hline BMI $(Z \text {-score })^{\star}$ & $2 \mathrm{SN} / 21 \mathrm{NW} / 2 \mathrm{OB}$ & - & $1 \mathrm{SN} / 17 \mathrm{NW} / 4 \mathrm{EW} / 3 \mathrm{OB}$ & - \\
\hline $\mathrm{HbA1c}(\%)$ & 8.94 & 1.69 & - & - \\
\hline Disease duration (years) & $7 \cdot 0$ & $5 \cdot 7$ & - & - \\
\hline Folates $(\mathrm{ng} / \mathrm{ml})$ & $(17.4)$ & $(9 \cdot 6)^{\mathrm{a}}$ & $(19.9)$ & $(5 \cdot 1)^{\mathrm{a}}$ \\
\hline Homocysteine $(\mu \mathrm{mol} / \mathrm{l})$ & $(4 \cdot 8)$ & $(1 \cdot 1)^{\mathrm{a}}$ & $(7 \cdot 3)$ & $(1.4)^{b}$ \\
\hline TNF- $a$ protein $(\mathrm{pg} / \mathrm{ml})$ & 4.04 & $2.04^{a}$ & 2.69 & $1.69^{b}$ \\
\hline
\end{tabular}

T1D, type 1 diabetes; F, female; M, male; SN, subnutrition; NW, normal weight; EW, excess weight; OB, obesity; HbA1c, glycosylated Hb.

a,b Mean values with unlike superscript letters were significantly different between groups of at least $(P<0.05)$.

${ }^{*} \mathrm{SN}, Z$-score $<-2$; NW, Z-score $\geq-1$ and $\leq+1$; EW, Z-score $>+1$; OB, Z-score $>+2$.

We recently described, in Chilean T1D patients, lower plasmatic Hcy and higher serum TNF- $\alpha$ concentrations. We also observed a lower status of global DNA methylation in T1D patients, which was associated with serum folate concentration but not with Hcy. We found that at high folate concentration T1D patients had a lower global DNA methylation status compared with control subjects, which indicates that the presence of the disease determined the way the patient responds to different folate concentrations ${ }^{(17)}$. To the authors knowledge, there are no published data about the TNFa gene promoter methylation status in T1D patients without complications, even though it is a relevant molecule in the pathogenesis of this disease. On the basis of what is stated above, we hypothesised that T1D patients had hypomethylation in the TNFa gene promoter and that this pattern was associated with Hcy, folate and TNF- $\alpha$ concentrations in blood. We wanted to determine the percentage of TNFa gene promoter methylation and its relationship with blood levels of TNF- $\alpha$, Hcy and folates in T1D patients, in order to determine whether a distinct methylation pattern is present in T1D patients with the purpose of distinguishing a potential novel biomarker for T1D.

\section{Methods}

\section{Study subjects}

As seen in Table 1, twenty-five T1D patients were recruited from the Institute of Maternal and Child Research of the University of Chile, and from the Adult Endocrinology Service, both at the San Borja Arriarán Hospital (Santiago, Chile). A sample of twenty-five patients and twenty-five controls was chosen because it returned a statistical power of $80 \%$ to determine differences in the variables studied with a CI of $95 \%$. Written informed consent was obtained from all patients ( $>10$ years) and/or their parents or tutors ( $<10$ years), and from all control subjects. T1D diagnosis was performed based on the ADA criteria. A total of twenty-five healthy subjects were recruited from the School of Medicine and from the Institute of Nutrition and Food Technology of the University of Chile.
Control subjects did not have T1D and/or other autoimmune diseases or direct family history of these conditions. Because T1D incidence decreases in adulthood, after the age of 14 years $^{(18)}$, all control subjects were $>20$ years old to have the certainty that this population had the lowest probability of developing T1D.

This study was conducted according to the guidelines laid down in the Declaration of Helsinki, and all procedures involving human patients were approved by the Ethical Committee of the School of Medicine of the University of Chile and by the Ethical Committee of the Central Health Service (San Borja Arriarán Hospital).

\section{Sample collection}

Samples were collected after a 12-h fast by venepuncture, and were centrifuged at $4000 \mathrm{rpm}$ for $5 \mathrm{~min}$, within $1 \mathrm{~h}$ from collection. We separated serum, plasma and whole blood with EDTA for further analyses. We measured glycosylated $\mathrm{Hb}$ (HbA1c) levels in the serum of T1D patients, by the "Tina-Quant ${ }^{\circledR,}$ II immunoturbidimetric method ${ }^{(19)}$ in San Borja Arriarán Hospital's Central Laboratory, as a reference of the metabolic control in these subjects.

\section{Homocysteine, folate and TNF- $\alpha$ quantification in blood}

Total Hcy was quantified by an enzyme conversion immunoassay ${ }^{(20)}$ and folates were measured using liquid chromatography with tandem $\mathrm{MS}^{(21)}$. Both assays were performed by an external certified laboratory (Red Lab Clinical Laboratory). TNF- $\alpha$ quantification was conducted by a Luminex ${ }^{\circledR}$ test kit (Human Adipokine Magnetic Bead Panel 2; EMD Millipore Corporation), as previously described ${ }^{(17)}$.

\section{DNA extraction and treatment}

DNA was extracted from leucocytes present in the whole blood with EDTA. The extraction was performed after sample collection, and the DNA obtained was stored refrigerated $\left(2-4^{\circ} \mathrm{C}\right)$ 
Epigenetics in type 1 diabetes

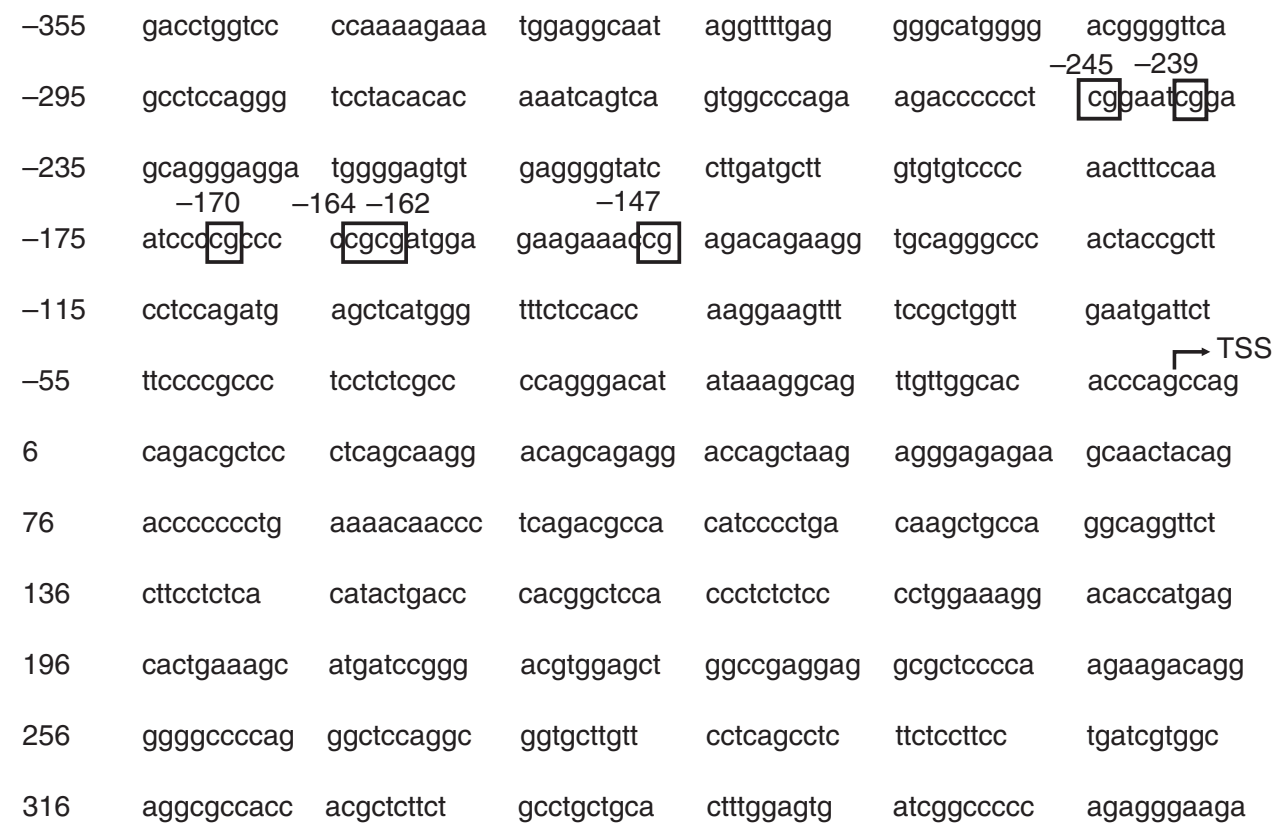

Fig. 1. TNFa gene promoter region upstream the transcription start site (TSS). Six CpG were considered in this analysis, which are numbered in the figure according to their distance to the TSS. The TSS was determined according to Economou ${ }^{(30)}$. Ensembl version: ENSG00000232810.

according to the manufacturer's instructions. The Wizard Genomic DNA Purification kit (Promega ${ }^{\circledR}$ ) was used for this purpose.

The extracted DNA was then modified with sodium bisulfite with the methylamp DNA modification kit (Epigentek $^{\circledR}$ ), according to the manufacturer's instructions. After denaturation of DNA and a 90-min incubation with sodium bisulfite, nonmethylated cytosines are converted to uracil. Modified DNA was stored at $-20^{\circ} \mathrm{C}$, as indicated by the manufacturer.

\section{TNFa gene expression}

A subsample of both control and patient groups ( $n$ 6) was selected randomly with the purpose of studying TNFa gene expression. RNA was extracted from leucocytes present in whole blood using TRIzol ${ }^{\circledR}$ Reagent (Thermo Fisher Scientific) according to the manufacturer's instructions. Total RNA was treated with DNAse with DNAfree kit (Ambion) and converted to first-strand complementary DNA (cDNA) with Moloney murine leukaemia virus (M-MLV) RT, utilising random hexamers (Invitrogen) and deoxynucleotide phosphate (dNTP) mix (Bioline) according to the manufacturer's instructions. The resultant cDNA was amplified using TaqMan probes (TaqMan ${ }^{\circledR}$ Hs01113624_g1; Thermo Fisher Scientific). Real-time PCR was performed in a Stratagene $\mathrm{M} \times 3000$ ' system (Agilent Technologies) according to the manufacturer's recommendations. The TNFa gene's relative expression was normalised with $\beta$-actin (Applied Biosystems), selected as an internal control. Fold change between groups was calculated by the $2^{-\Delta \Delta C_{t}}$ method.

\section{TNFa gene methylation assay}

We then quantified the percentage of methylation in the $T N F a$ gene promoter using specific primers for modified DNA, by real-time $\mathrm{PCR}$ (in a $\mathrm{M} \times 3000 \mathrm{P}$ system; Agilent Technologies). Then, we analysed the melting curves as previously described $^{(22)}$. This method allows the quantification of the methylation percentage in the selected DNA fragment.

Primers were designed by using the MethPrimer web tool (http://www.urogene.org/methprimer/) for TNFa gene promoter amplification. These were designed to only recognise bisulfite-treated DNA, increasing the specificity of this technique. We obtained a 147-bp amplicon containing six CpG islands (CGI) (Fig. 1). TNF- $\alpha$ forward primer AGT TAGTGG TTT AGA AGA TTT TTT T and TNF- $\alpha$ reverse primer ATA AAC CCT ACA CCT TCT ATC TC were utilised. We used $12.5 \mu$ of Brilliant II SYBR Green QPCR Master Mix (Stratagen; Agilent Technologies) for each sample, according to the supplier's indications.

For the real-time PCR, we used 40 ng of modified DNA and we test each sample in duplicate. We conducted an initial denaturation step at $95^{\circ} \mathrm{C}$ for $10 \mathrm{~min}$, followed by $15 \mathrm{~s}$ at $95^{\circ} \mathrm{C}$. The annealing phase was carried out at $50^{\circ} \mathrm{C}$ for $15 \mathrm{~s}$ for forty cycles, and we conducted a final extension step at $72^{\circ} \mathrm{C}$ for $15 \mathrm{~s}$. The melt of the PCR product was performed from 60 to $90^{\circ} \mathrm{C}$, rising in $0.5^{\circ} \mathrm{C}$ increments, waiting $5 \mathrm{~s}$ at each step, and acquiring fluorescence at each temperature increment, as previously described ${ }^{(22)}$.

We performed a standard curve (Fig. 2(a) and (b)) to calculate the methylation percentage of the TNFa gene promoter, using $5 '$ methylated DNA concentrations (0, 25, 50, 75 and $100 \%$ methylation). For this purpose, we used a $100 \%$ methylated control human DNA and a 100\% non-methylated control human DNA (EpiTect ${ }^{\circledR}$ PCR Control DNA; Qiagen).

\section{Statistical analysis}

We conducted a Shapiro-Wilk normality test to evaluate the variable distribution in both the T1D and the control groups. 


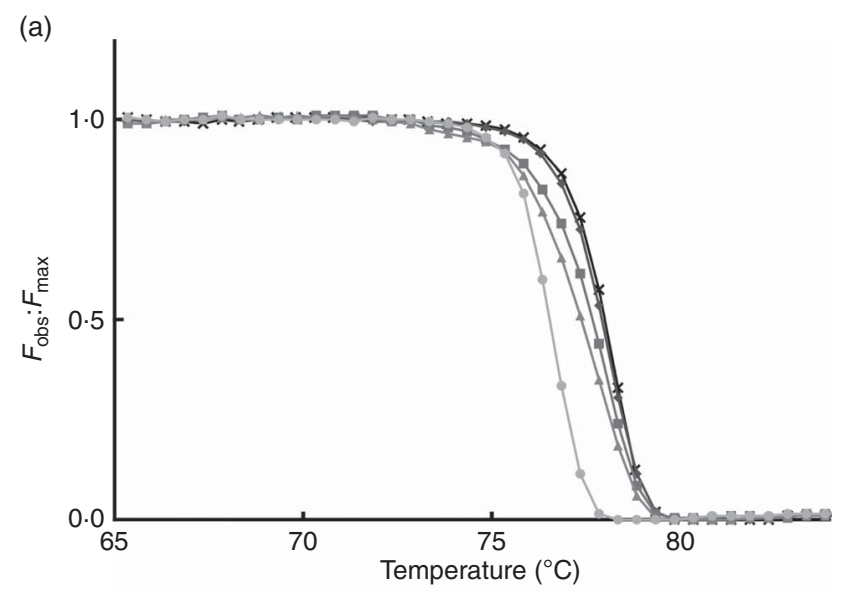

(b)

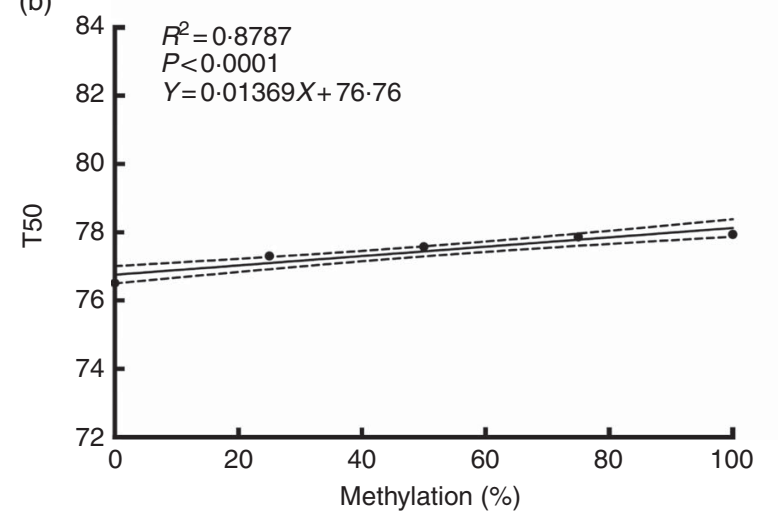

Fig. 2. Normalised fluorescence curve (a) by $F_{\text {obs }}: F_{\max }$ ratio $\left(F_{\max }\right.$ is a theoretical fluorescence for each temperature point that could be obtained if DNA melting occurs linearly with a constant slope). From this curve, we can obtain T50 (temperature at which half of the DNA is melted and half is forming a double strand). At higher levels of DNA methylation, the normalised fluorescence curve is positioned at a higher temperature as seen in the graph. Standard curve (b) is used for interpolation of the TNFa gene promoter methylation percentage; T50 shows a positive correlation with the percentage of methylation in the sample. $F_{\text {obs }}$, observed fluorescence; $F_{\max }$, maximum fluorescence; T50, temperature 50; - - $0 \%$;,$- \quad 25 \%$; ,$- 50 \% ; \multimap, 75 \% ; \multimap, 100 \%$.

To determine statistical differences between groups, a Student's $t$ test was performed for parametric variables and a MannWhitney $U$ test for non-parametric variables. We subdivided both groups into three subgroups according to age. (Age groups were defined by separating the sample in tertiles. Age groups in patients: $<10$ years $(n 9), 10-15$ years $(n 8)$ and $<15$ years $(n 8)$. Age groups in controls: $<27$ years $(n 9), 28-30$ years $(n 8)$ and $>30$ years $(n 8)$.) We used one-way ANOVA and Tukey's post hoc for parametric variables and Kruskal-Wallis test and Dunn's post hoc for non-parametric variables, to find any differences between these subgroups. To determine any association between variables, we used Pearson's correlation test for parametric variables and Spearman's test for non-parametric variables. Results are presented as means and standard deviations for parametric variables and as median (interquartile range) for non-parametric variables. We used a significance level of $P<0 \cdot 05$. GraphPad Prism 6.0 software (GraphPad Software, Inc.) was used for the statistical analysis.

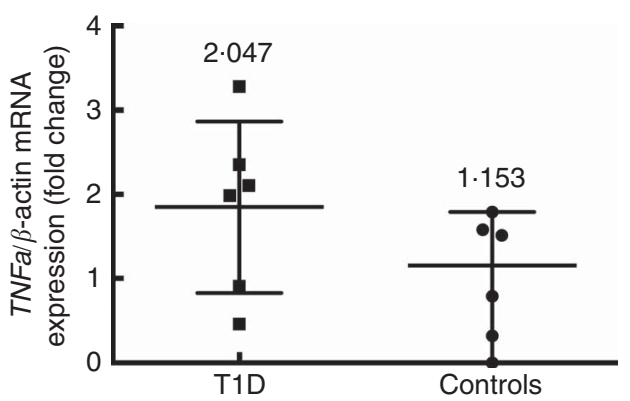

Fig. 3. TNFa gene expression in control subjects $(n 6)$ and T1D patients $(n 6)$. Relative TNF-a mRNA expression was calculated with the $2^{\Delta \Delta C_{t}}$ method and normalised with $\beta$-actin as housekeeping. Values are medians and interquartile ranges.

\section{Results \\ Clinical and diagnostic characteristics}

Table 1 shows the characteristics of both groups studied. Both T1D patients and control subjects had blood Hcy concentrations within the reference values $(4.44-13.56 \mu \mathrm{mol} / 1$ in females and $5 \cdot 46-16.20 \mu \mathrm{mol} / 1$ in males) and folate concentrations within the reference values $(>5.38 \mathrm{ng} / \mathrm{ml})$. We did not find any significant difference in folate serum concentrations between groups. Hcy plasma concentrations were lower in patients than in controls. We found higher TNF- $\alpha$ protein concentrations in blood from T1D patients in comparison with control subjects. We did not find any differences in TNF- $\alpha$ protein concentrations in blood, by age or sex, in patients or controls (data not shown).

\section{Gene expression and methylation status}

Six patients and six control subjects were selected randomly to analyse the TNFa gene expression (Fig. 3). Despite the small number of subjects included in this assay, a tendency towards a higher expression in patients (median 2.047 (interquartile range $2 \cdot 816)$ ) than in controls (median 1.153 (interquartile range 1.791)) $(P=0.093)$ is observed. This is in the same direction of what was seen with the concentration of TNF- $\alpha$ protein in blood. The TNFa gene promoter methylation status was higher in T1D patients $(39.2$ (SD 19.5)\%) than in controls (25.4 (SD $13.7) \%)(P=0.008)$ (Fig. 4(a)), and we did not find any significant difference according to age for the TNFa gene promoter methylation status in T1D patients $(P=0.589)$ (Fig. 4(b)) or in control subjects $(P=0.536)$.

\section{Correlations}

We found that the TNFa gene promoter methylation status was positively associated with Hcy concentration in T1D patients (Fig. 5) $(r$ 0.555, $P=0.007)$ but not in controls $(r-0.122$, $P=0 \cdot 872)$.

\section{Discussion}

The TNFa gene promoter does not have a CGI but has a region rich in $\mathrm{CpG}^{(12)}$. (According to Takai \& Jones ${ }^{(23)}$ a CGI 

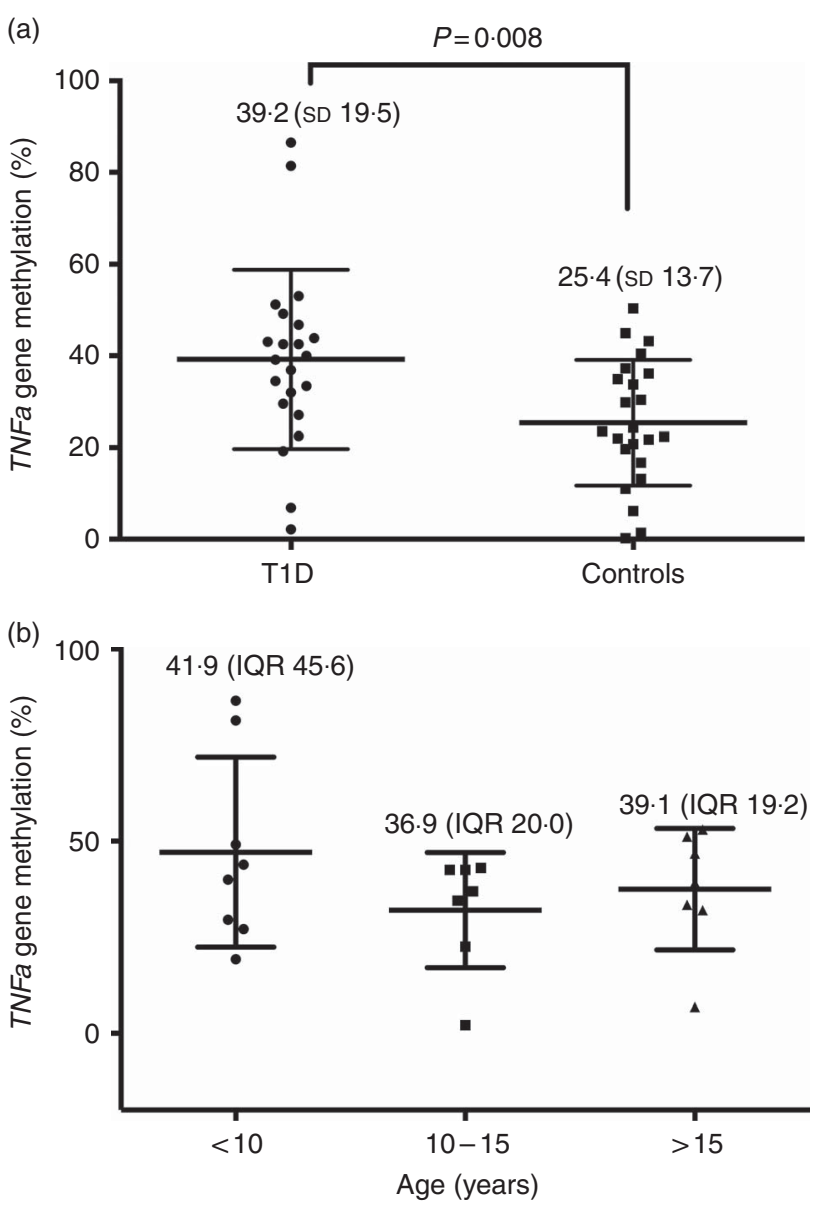

Fig. 4. Methylation status (a) of the TNFa gene promoter in type 1 diabetes (T1D) patients ( $n$ 22) and control subjects ( $n$ 23), and its variation according to age in T1D patients (b). (a) Values are means and standard deviations (Student's $t$ test). (b) Values are medians and interquartile ranges (IQR). Age groups $-<10$ years $(n 9) ; 10-15$ years $(n 8) ;>15$ years $(n 8)$ (Kruskal-Wallis test and Dunn's post hoc).

corresponds to a DNA region with $>500$ bp with a CG content $>55 \%$ and an observed:expected C:G ratio $>65 \%$ ). A correlation has been demonstrated between the methylation statuses of genes not containing CGI at their $5^{\prime}$ end and their mRNA, which indicates that the expression control of these genes with a low CpG density is also a subject of epigenetic regulation ${ }^{(24)}$.

To date, there are only a few studies about TNFa gene methylation status in several conditions. Hermsdorff et al. ${ }^{(16)}$ determined that the methylation status of $2 \mathrm{CpG}$ sites in the TNFa gene promoter (numbered +217 and +327 in our sequence) was inversely correlated with TNF- $\alpha$ blood concentrations and with truncal fat levels in normal weight women. Kojima et $a l .{ }^{(25)}$ found higher methylation rates in seven CpG motifs (numbered $-303,-164,-120,-73,-50,-39$ and +10 in our sequence) in Japanese Rheumatoid Arthritis, which seems to be a distinctive feature of the disease. Campion et al. ${ }^{(14)}$ found higher levels of methylation in $12 \mathrm{CpG}$ (numbered -304, $-245,-239,-170,-164,-162,-147,-120,-73,-50,-39$ and +10 in our sequence) in the $T N F a$ gene promoter in obese subjects who responded to a low energetic diet, in comparison with those who did not respond. They also found a positive

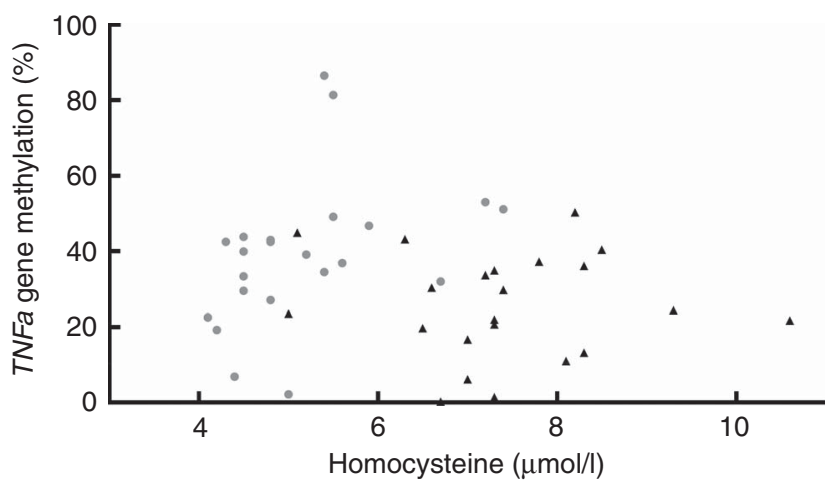

Fig. 5. Blood homocysteine and TNFa gene promoter methylation status. Positive association between homocysteine levels and TNFa gene promoter methylation (\%) in type 1 diabetes (T1D) patients (O, TID: $P 0.007, r 0.555$ ), and no association between these parameters in control subjects $(\boldsymbol{\Lambda}, P 0.872$, $r-0.1219, R^{2}$ 0.015). T1D: Spearman's test; Controls: Pearson's test.

association between total methylation level and the initial concentration of TNF- $\alpha$ in the study subjects. In all these studies, the authors measured each CpG methylation and correlated each status to TNF- $\alpha$ levels. We measured the methylation status of a region of the promoter, which includes 6 CpG $(-245,-239,-170,-164,-162,-147)$, but we did not measure the methylation status of each CpG. We chose this region because it was considered in all the studies mentioned above, being the only ones evoked to study this epigenomic area.

On the other hand, there are only a few studies about genespecific methylation status in T1D. There was one work on latent autoimmune diabetes in adults, which determined a higher methylation status in the Forkhead box P3 (FOXP3) gene in $\mathrm{T}$ CD4+ lymphocytes with a higher expression of such gene $^{(26)}$. In T1D, nineteen CPG have been related with the risk of development of diabetic nephropathy, and some of them have a different methylation status relative to control subjects (T1D patients without nephropathy) ${ }^{(27)}$. A recent study showed differences in the methylation status of $349 \mathrm{CpG}$, which represent 233 genes, including TNF- $\alpha$, in patients with or without proliferative diabetic retinopathy ${ }^{(28)}$. The authors suggest that these epigenetic changes may be useful to predict the development of this diabetic complication.

To our knowledge, this is the first study to report the methylation status of the TNFa gene promoter in T1D patients without disease complications. Our results shows that T1D patients have higher TNF- $\alpha$ protein blood levels, a tendency towards higher TNFa gene expression but also higher methylation levels in the TNFa gene promoter region studied. However, we did not find any association between these parameters. The lack of association between TNFa gene promoter methylation and the expression of TNFa gene could be explained by the fact that several transcription-factor-binding sites have been identified in the TNFa gene promoter, included in the region studied here ${ }^{(29,30)}$. The methylation status of this region could modify the binding of these transcription factors, which may reflect the variability in the regulation of the expression of this gene ${ }^{(31-33)}$. Although it is known that, in general terms, higher methylation is related with gene silencing, 
some exceptions could be encountered. It has been found that the inactive $\mathrm{X}$ chromosome is less methylated than the active one ${ }^{(34)}$. Therefore, it could be difficult to predict a direct relationship between methylation status and gene expression. It is worth mentioning that besides monocytes/ macrophages TNF- $\alpha$ is produced by several cell types including smooth and cardiac muscle cells, fibroblasts, osteoclasts and many others ${ }^{(35)}$. Therefore, the differences observed between the concentrations of this protein in blood, in both groups studied, could be related to the production of this cytokine by all these other cells.

Leucocytes were chosen, instead of for example $\beta$-cells, because they are easy to obtain from a blood sample (a quick and non-invasive method). In addition, in several publications it has been demonstrated that they are a good source of prediction of a disease state, because they respond to diverse metabolic and/or pathological conditions through the regulation of the expression of different genes involved in the immune ${ }^{(36)}$ and inflammatory ${ }^{(37)}$ response. Even though the epigenetic modification examined only in leucocytes could not completely explain the serum levels of $\mathrm{TNF}-\alpha$, considering that cells actively producing this cytokine could be found in tissues in addition to being in the circulation, this could be a first approach to noticing differences between T1D patients and control subjects.

By design, we included only 20-year-old healthy subjects as part of the control group. The purpose of this was to have the certainty that these subjects had the lowest probability of developing T1D, in case the difference in the methylation status of TNFa gene was present before the onset of the disease. We previously reported that global DNA methylation was modified in T1D patients and control subjects according to age ${ }^{(17)}$, but we did not find any significant difference according to age in the TNFa gene promoter methylation status in T1D patients or control subjects. This is in agreement with the study by Eckhardt $e$ t $a l .{ }^{(24)}$, where the authors compared the methylation percentage of 1.9 million CpG (from different tissues and cells from chromosomes 6, 20 and 22) in two age groups (26 and 68 years on average, respectively), and did not find differences between groups. This indicates that probably gene-specific methylation is less affected by age than global DNA methylation. They did find differences between the different tissues and cells studied, demonstrating that gene methylation is tissue and cell specific, which corresponds with the work of Lokk et $a l^{(38)}$. Hence, the study of gene-specific methylation could be a better way to approach the search for biomarkers of early genetic risk. Even though Gowers et $a l^{(39)}$ found that the methylation in TNFa gene promoter CpG sites $-304,-245$ and -239 decreases with age in healthy subjects, we did not observe any tendency in that direction in our study or control group, but our age range was narrower.

The association that we found between Hcy levels and TNFa gene promoter methylation status could be a consequence of the alteration in Hcy metabolism in T1D. Two independent Hcy remethylation pathways have been described so far. The first one, dependent on folate, is catalysed by the methionine synthase (MS) enzyme, which takes a methyl group from 5-methyl tetrahydrofolate. The second, independent of folate, is catalysed by the betaine-homocysteine methyltransferase
(BHMT) enzyme, which takes a methyl group from betaine, a product of choline oxidation. The other pathway involved in the metabolism of Hcy is its trans-sulfuration, which results in the irreversible transformation of Hcy into cystathionine catalysed by the cystathionine- $\beta$-synthase (CBS) enzyme, and then the cystathionine is transformed into cysteine by the action of cysteine- $\gamma$-lyase $(\mathrm{CGL})^{(40)}$. There are two methyltransferases that play an important role in this process - phosphatidylethanolamine $N$-methyltransferase (PEMT) and glycine $N$-methyltransferase - both of which regulate the equilibrium between SAM and $S$-adenosylhomocysteine ${ }^{(41)}$.

Insulin levels are capable of modulating the activity of the enzymes named above, and this metabolic impairment could have an impact on gene-specific methylation status, as well as on global DNA methylation status, which agrees with several studies: Jacobs et al. $^{(42)}$ demonstrated in T1D rats a higher activity of CBS and CGL; Nieman \& Schalinske ${ }^{(43)}$ found higher hepatic activity of GNMP and PEMT and a decrease in MS activity, accompanied by a lower global DNA methylation status and a negative correlation between blood glucose and Hcy, in T1D. In both experiments, these modifications were reversed by exogenous insulin administration A higher activity in the trans-sulfuration pathway was also observed in T1D humans without insulin treatment ${ }^{(44)}$. Williams \& Schalinske ${ }^{(45)}$ found higher BHMT activity and lower MS activity and global DNA methylation status in T1D rats. It is relevant to point out that, when our study was conducted, only two T1D patients had HbA1c levels under $7 \%$, which indicates that the majority of our patients had a poor metabolic control, which could be related to lower insulin levels and/or chronic hyperglycaemia, both having an impact on Hcy metabolism and therefore on global and specific DNA methylation patterns.

We chose the analysis of melting curves to assess the methylation of the TNFa gene promoter, because it is a quantitative and sensitive technique that can be performed in any real-time PCR equipment capable of measuring fluorescence, and is easy to implement. This is because a higher methylated DNA, after bisulfite treatment, retains more cytosines and therefore has a higher melting temperature compared with an unmethylated DNA ${ }^{(46)}$. It has been proven that the percentage of methylation of a selected DNA region has a positive linear correlation with the temperature at which $50 \%$ of the sample melts and $50 \%$ forms double strands, and therefore when the fluorescence has descended to half of the initial value ${ }^{(22)}$; this enables us to create a standard curve to calculate the methylation percentage of our samples, using methylated and unmethylated DNA controls.

To our knowledge, this is the first study to report the methylation status of the TNFa gene promoter in T1D Chilean patients without complications, and to show differences relative to control healthy subjects. Considering that TNF- $\alpha$ is an important cytokine in the pathogenesis of T1D and its complications, it is necessary to conduct more studies to determine whether this differential methylation status is due to an alteration in Hcy metabolism because of the disease or whether it is present before the onset of this autoimmune disease, in which case it could be postulated as a possible biomarker of early risk. 


\section{Acknowledgements}

The authors thank all participants for their cooperation.

This project was supported by FONDECYT grant no. 1130240.

In brief, the contribution to this article of each author is as follows: V. A.-J., experimental design, experimental procedures, data analysis, manuscript drafting and manuscript revision; D. F. G.-D., data analysis and manuscript revision; E. C., manuscript revision and F. P.-B., experimental design, data analysis, manuscript preparation and manuscript revision.

The authors declare that there are no conflicts of interest.

\section{References}

1. Pugliese A (2013) The multiple origins of type 1 diabetes. Diabet Med 30, 135-146.

2. Redondo MJ \& Eisenbarth GS (2002) Genetic control of autoimmunity in type I diabetes and associated disorders. Diabetologia 45, 605-622.

3. Adeghate E, Schattner P \& Dunn E (2006) An update on the etiology and epidemiology of diabetes mellitus. Ann N Y Acad Sci 1084, 1-29.

4. Hermann R, Knip M, Veijola R, et al. (2003) Temporal changes in the frequencies of HLA genotypes in patients with type 1 diabetes - indication of an increased environmental pressure? Diabetologia 46, 420-425.

5. Fourlanos S, Varney MD, Tait BD, et al. (2008) The rising incidence of type 1 diabetes is accounted for by cases with lower-risk human leukocyte antigen genotypes. Diabetes Care 31, 1546-1549.

6. Hewagama A \& Richardson B (2009) The genetics and epigenetics of autoimmune diseases. J Autoimmun 33, 3-11.

7. Anderson OS, Sant KE \& Dolinoy DC (2012) Nutrition and epigenetics: an interplay of dietary methyl donors, one-carbon metabolism and DNA methylation. J Nutr Biochem 23, 853-859.

8. Nazki FH, Sameer AS \& Ganaie BA (2014) Folate: metabolism, genes, polymorphisms and the associated diseases. Gene $\mathbf{5 3 3}$, $11-20$.

9. Devaraj S, Glaser N, Griffen S, et al. (2006) Increased monocytic activity and biomarkers of inflammation in patients with type 1 diabetes. Diabetes 55, 774-779.

10. Grunnet LG, Aikin R, Tonnesen MF, et al. (2009) Proinflammatory cytokines activate the intrinsic apoptotic pathway in beta-cells. Diabetes 58, 1807-1815.

11. Rabinovitch A \& Suarez-Pinzon WL (1998) Cytokines and their roles in pancreatic islet beta-cell destruction and insulindependent diabetes mellitus. Biochem Pharmacol 55, 1139-1149.

12. Sullivan KE, Reddy AB, Dietzmann K, et al. (2007) Epigenetic regulation of tumor necrosis factor alpha. $\mathrm{Mol}$ Cell Biol 27, 5147-5160.

13. Sharples AP, Polydorou I, Hughes DC, et al. (2016) Skeletal muscle cells possess a 'memory' of acute early life TNF-alpha exposure: role of epigenetic adaptation. Biogerontology 17, 603-617.

14. Campion J, Milagro FI, Goyenechea E, et al. (2009) TNF-alpha promoter methylation as a predictive biomarker for weightloss response. Obesity (Silver Spring) 17, 1293-1297.

15. Gomez-Uriz AM, Goyenechea E, Campion J, et al. (2014) Epigenetic patterns of two gene promoters (TNF-alpha and $\mathrm{PON})$ in stroke considering obesity condition and dietary intake. J Physiol Biochem 70, 603-614.
16. Hermsdorff HH, Mansego ML, Campion J, et al. (2013) TNF-alpha promoter methylation in peripheral white blood cells: relationship with circulating TNFalpha, truncal fat and n-6 PUFA intake in young women. Cytokine 64 , $265-271$.

17. Arroyo-Jousse V, Garcia-Diaz DF \& Perez-Bravo F (2015) [Global DNA methylation and homocysteine levels are lower in type 1 diabetes patients]. Rev Med Chil 143, 562-568.

18. Diaz-Valencia PA, Bougneres P \& Valleron AJ (2015) Global epidemiology of type 1 diabetes in young adults and adults: a systematic review. BMC Public Health 15, 255.

19. Jones TG, Warber KD \& Roberts BD (2010) Analysis of hemoglobin A1c from dried blood spot samples with the Tina-quantR II immunoturbidimetric method. J Diabetes Sci Technol 4, 244-249.

20. Frantzen F, Faaren AL, Alfheim I, et al. (1998) Enzyme conversion immunoassay for determining total homocysteine in plasma or serum. Clin Chem 44, 311-316.

21. Owens JE, Holstege DM \& Clifford AJ (2005) Quantitation of total folate in whole blood using LC-MS/MS. J Agric Food Chem 53, 7390-7394.

22. Smith E, Jones ME \& Drew PA (2009) Quantitation of DNA methylation by melt curve analysis. BMC Cancer $\mathbf{9}, 123$.

23. Takai D \& Jones PA (2002) Comprehensive analysis of CpG islands in human chromosomes 21 and 22. Proc Natl Acad Sci U S A 99, 3740-3745.

24. Eckhardt F, Lewin J, Cortese R, et al. (2006) DNA methylation profiling of human chromosomes 6, 20 and 22. Nat Genet 38, 1378-1385.

25. Kojima A, Kobayashi T, Ito S, et al. (2015) Tumor necrosis factor-alpha gene promoter methylation in Japanese adults with chronic periodontitis and rheumatoid arthritis. $J$ Periodontal Res 51, 350-358.

26. Li Y, Zhao M, Hou C, et al. (2011) Abnormal DNA methylation in CD4+ T cells from people with latent autoimmune diabetes in adults. Diabetes Res Clin Pract 94, 242-248.

27. Bell CG, Teschendorff AE, Rakyan VK, et al. (2010) Genomewide DNA methylation analysis for diabetic nephropathy in type 1 diabetes mellitus. BMC Med Genomics 3, 33 .

28. Agardh E, Lundstig A, Perfilyev A, et al. (2015) Genome-wide analysis of DNA methylation in subjects with type 1 diabetes identifies epigenetic modifications associated with proliferative diabetic retinopathy. BMC Med 13, 182.

29. Kramer B, Meichle A, Hensel G, et al. (1994) Characterization of an Krox-24/Egr-1-responsive element in the human tumor necrosis factor promoter. Biochim Biophys Acta 1219, 413-421.

30. Economou JS, Rhoades K, Essner R, et al. (1989) Genetic analysis of the human tumor necrosis factor alpha/cachectin promoter region in a macrophage cell line. J Exp Med 170, 321-326.

31. Comb M \& Goodman HM (1990) CpG methylation inhibits proenkephalin gene expression and binding of the transcription factor AP-2. Nucleic Acids Res 18, 3975-3982.

32. Clark SJ, Harrison J \& Molloy PL (1997) Sp1 binding is inhibited by $(\mathrm{m}) \mathrm{Cp}(\mathrm{m}) \mathrm{CpG}$ methylation. Gene 195, 67-71.

33. Iguchi-Ariga SM \& Schaffner W (1989) CPG methylation of the cAMP-responsive enhancer/promoter sequence TGACGTCA abolishes specific factor binding as well as transcriptional activation. Genes Dev 3, 612-619.

34. Weber M, Davies JJ, Wittig D, et al. (2005) Chromosome-wide and promoter-specific analyses identify sites of differential DNA methylation in normal and transformed human cells. Nat Genet 37, 853-862.

35. Zelova H \& Hosek J (2013) TNF-alpha signalling and inflammation: interactions between old acquaintances. Inflamm Res 62, 641-651. 
36. Ma J, Dempsey AA, Stamatiou D, et al. (2007) Identifying leukocyte gene expression patterns associated with plasma lipid levels in human subjects. Atherosclerosis 191, 63-72.

37. Wettinger SB, Doggen CJ, Spek CA, et al. (2005) High throughput mRNA profiling highlights associations between myocardial infarction and aberrant expression of inflammatory molecules in blood cells. Blood 105, 2000-2006.

38. Lokk K, Modhukur V, Rajashekar B, et al. (2014) DNA methylome profiling of human tissues identifies global and tissue-specific methylation patterns. Genome Biol 15, 154 .

39. Gowers IR, Walters K, Kiss-Toth E, et al. (2011) Age-related loss of CPG methylation in the tumour necrosis factor promoter. Cytokine 56, 792-797.

40. Finkelstein JD (1998) The metabolism of homocysteine: pathways and regulation. Eur J Pediatr 157, Suppl. 2, S40-S44.

41. Williams KT \& Schalinske KL (2007) New insights into the regulation of methyl group and homocysteine metabolism. J Nutr 137, 311-314.
42. Jacobs RL, House JD, Brosnan ME, et al. (1998) Effects of streptozotocin-induced diabetes and of insulin treatment on homocysteine metabolism in the rat. Diabetes $\mathbf{4 7}$, 1967-1970.

43. Nieman KM \& Schalinske KL (2011) Insulin administration abrogates perturbation of methyl group and homocysteine metabolism in streptozotocin-treated type 1 diabetic rats. Am J Physiol Endocrinol Metab 301, E560-E565.

44. Abu-Lebdeh HS, Barazzoni R, Meek SE, et al. (2006) Effects of insulin deprivation and treatment on homocysteine metabolism in people with type 1 diabetes. J Clin Endocrinol Metab 91, 3344-3348.

45. Williams KT, Garrow TA \& Schalinske KL (2008) Type I diabetes leads to tissue-specific DNA hypomethylation in male rats. J Nutr 138, 2064-2069.

46. Kurdyukov S \& Bullock M (2016) DNA methylation analysis: choosing the right method. Biology (Basel) 5, 1-21. 